\title{
Community diabetes in South London
}

\author{
MARK CHAMLEY
}

\begin{abstract}
Lambeth is an inner-city London borough with an ethnically and economically diverse population with a relatively high prevalence of diabetes. Forty-eight GP surgeries are supported for secondary care by two large, renowned teaching hospitals. A major reorganisation of care was undertaken in 2011, with a shift towards a highly integrated approach to multidisciplinary care delivered wherever possible in the community setting (hospitals continued to care for people with type 1 diabetes, advanced diabetes compilations, or current or planned pregnancy). Structural barriers to care delivery (e.g. bureaucracy relating to referrals) were identified and overcome. The integrated model provides a range of initiatives to improve patients' self-care, including structured diabetes education. Educational sessions and 'virtual clinics' share experience and knowledge among healthcare professionals, and expert support is always available to GP practices. The last four years have seen an improved detection rate of diabetes locally, improved diabetes outcomes, a steady reduction in referrals to hospital diabetes clinics and a reduction in local admissions due to diabetes. Patient feedback is highly positive and the future of the service looks bright.
\end{abstract}

Br J Diabetes Vasc Dis 2015;15:78-81

Key words: diabetes, community care, integrated care, multidisciplinary care, structured education

\section{Community diabetes in south London}

Lambeth is an inner-city borough with a population of at least 310,000 . It covers an area stretching from the south bank of the River Thames to the suburbs of neighbouring Croydon and is one of the most deprived Boroughs in London. The demographics are hugely diverse, with large numbers of people of AfricanCaribbean and Portuguese heritage living locally and more than 100 languages spoken.

Partner, Crown Dale Medical Centre; Clinical Lead, Lambeth Diabetes Intermediate Care Team; Board Member, South East London Health Partnership, London, UK

Address for correspondence: Dr Mark Chamley Crown Dale Medical Centre, 61 Crown Dale, London, SE19 3NY, UK. E-mail: mark.chamley@nhs.net

http://dx.doi.org/10.15277/bjdvd.2015.013

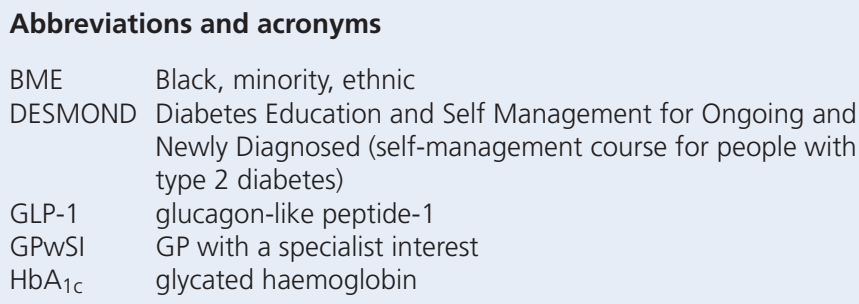

Based on GP registers, 15,200 people with diabetes live in Lambeth. Although the population is younger, on average, than most of the UK population, a high prevalence of diabetes is related to high levels of obesity and also the substantial BME population, who tend to develop type 2 diabetes at a younger age than some other ethnic groups.

There are 48 GP surgeries in Lambeth, grouped into three localities. Two large, renowned teaching hospitals provide secondary care: Guy's \& St Thomas' and King's College Hospital. Each has a large, academic and clinically cutting-edge diabetes unit.

In 2009, Healthcare for London published the "Diabetes Guide for London", which made a series of recommendations about how diabetes services should be provided in the capital. Some elements of the suggested model of care were already in place in Lambeth; however, local commissioners decided to undertake a major service redesign in 2011. A key element of this was a "shift" in care for people with type 2 diabetes from a hospital outpatient setting to specialist care in the community. These changes were facilitated by a $f 4$ million grant from the Guy's and St Thomas' Charity which funded a major change programme called the Diabetes Modernisation Initiative.

\section{The new service}

After the service redesign, the Lambeth Diabetes Intermediate Care Team was expanded with a new remit to directly provide patient care in community settings (Figure 1). Local commissioners and healthcare providers recognised that the traditional "hospital outpatient" model was not sustainable or affordable, with ever increasing numbers of people with type 2 diabetes. Another aim was to provide care for the person with diabetes closer to home and more integrated with the care provided by their GP.

It was important from the start that all involved were committed to making it work. Local diabetes consultants, the Intermediate Care Team and GPs agreed criteria for which patients should receive care in hospital, in the new community service and in primary care. The Diabetes Modernisation Initiative organised a series of patient participation events to seek service 
Figure 1. Two sites providing integrated local care in Lambeth

a) Team members at the community diabetes clinic at West Norwood Health and Leisure Centre

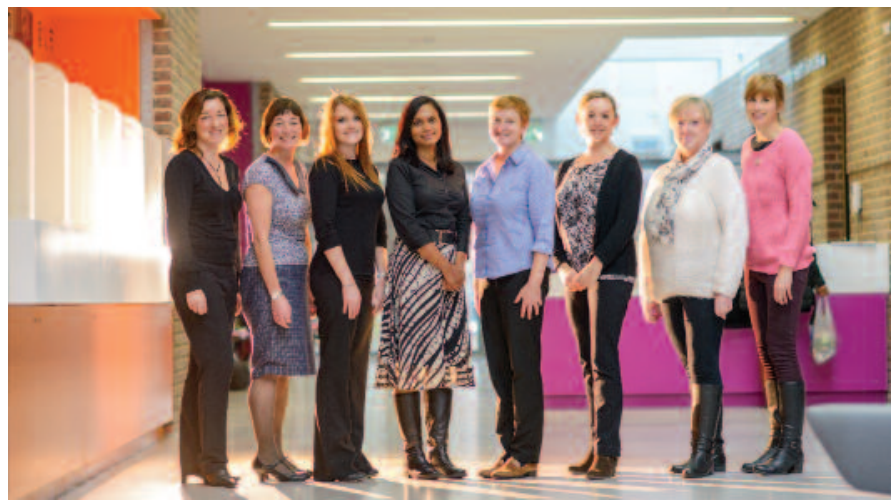

b) Gracefield Gardens Health Centre in Streatham

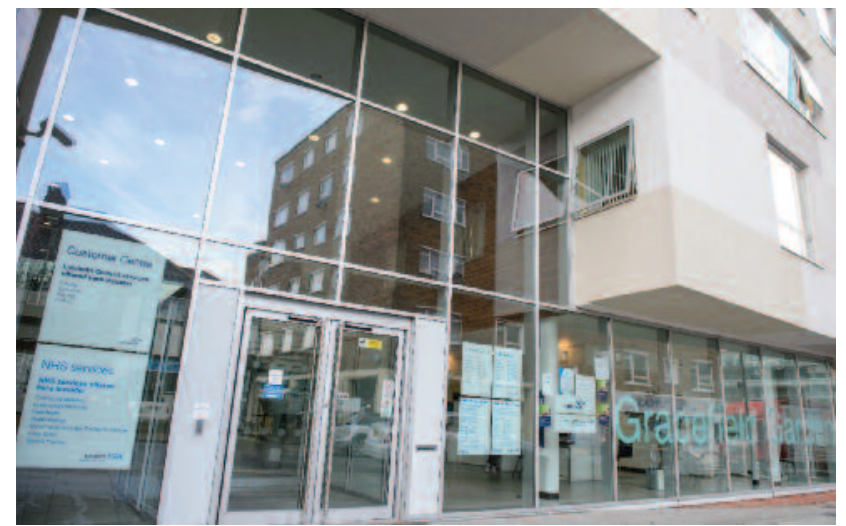

user input and approval for the changes being made. The strong message from local people with diabetes was that it did not matter where they received diabetes care: access to good quality care and a specialist, if needed, was important to them.

The referral criteria agreed were that the hospital-based services would provide care for people with type 1 diabetes, pre-conception and pregnancy care and for people with type 2 diabetes and advanced complications. For the vast majority of people with type 2 diabetes, GPs would refer to the new community-based service.

For the specialist diabetes units at St Thomas' and King's College Hospitals the new care model presented both challenges and opportunities. There was a planned shift away from secondary care services, which would result in a loss of income but better opportunities to integrate with the new community-based services. Opportunities included more capacity to see the most complex patients and also for hospital-based clinicians to work in the new community-based clinics.

The Diabetes Intermediate Care Team is unusual in that it is provided by a GP practice in Lambeth who were awarded a contract for the service in 2011. The clinical team is led by a GP specialist in diabetes and includes six diabetes specialist nurses, two specialist dieticians, three GPwSI, a pharmacist and healthcare assistants. There is considerable integration with the specialist hospital service: each community clinic is led by a hospital consultant and the dieticians are employed by the hospital trust and work in both secondary and community care. One diabetes nurse is on a hospital diabetes specialist nurse training programme, which includes a oneyear rotation into the community service.

Integrated working has been key to the success of the community service. Consultants are committed to providing clinical leadership and recognise that more and more diabetes care is moving into community settings. Whenever possible, we identify and overcome structural barriers to care related to healthcare provision: for example, it is straightforward to transfer care between the community service and secondary care without the need for formal referral letters. Many people with diabetes who have attended hospital follow-up for years are now being transferred to the community service.

The Diabetes Intermediate Care Team provides a range of services for people living with diabetes in Lambeth (Figure 2). The model of care developed includes a range of interventions designed to promote self-care for people with diabetes and to improve the quality of diabetes care provided by local GP practices.

\section{Community-based specialist diabetes clinics}

In 2011 the team were initially commissioned to provide two clinics per week, since expanded to four per week to meet increased numbers of referrals. Each operates in a different location in Lambeth and provides a hub for specialist diabetes care

Figure 2. Lambeth Diabetes Intermediate Care Team integrated care model around the person with diabetes and GPs

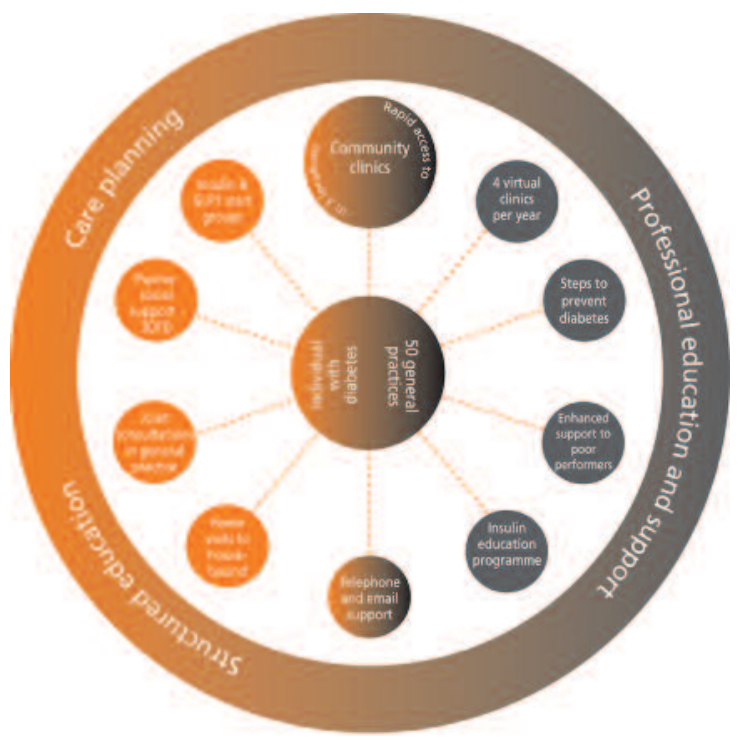


for nearby GPs. Patients will usually attend the clinic nearest to home, but can attend any clinic. Each clinic has six new patient appointments and 12 follow-up appointments. In 2014, 2,592 people were seen in the Lambeth Community Diabetes Clinics.

Appointments in the community clinics are truly multidisciplinary. New patients are invariably seen by a doctor, dietician and diabetes nurse. The healthcare assistant performs near-patient $\mathrm{HbA}_{1 c}$ testing. All patients attending the clinic are discussed in a meeting before the clinic starts so that the team can share thoughts on management options. The team meets again at the end of the clinic and all patients are presented in turn. Patients referred by their GP practice are generally seen for 3-4 appointments over six months and then discharged back to GP care. One of the reasons for the post-clinic meeting is to challenge colleagues about whether a patient needs to remain in the service or can be appropriately managed in primary care. All patients attending the community clinics are asked to complete a feedback questionnaire and the results have been overwhelmingly positive.

\section{Targeted support to general practices}

Each year, 10 Lambeth practices are provided with enhanced support from the Diabetes Intermediate Care Team. These practices usually reported the lowest outcome measures for $\mathrm{HbA}_{1 \mathrm{c}}$, blood pressure and cholesterol targets in the previous year, but sometimes received additional support for other reasons, such as the loss from the practice of a highly-trained nurse or GP. The enhanced support consists largely of a regular joint diabetes clinic at the practice, provided by a specialist nurse from the Diabetes Intermediate Care Team, with a practice nurse or GP sitting in. The aim is to review patients who are more complex and would otherwise need to be referred, but also to provide education and improved diabetes knowledge to the practice nurse or GP observing. This model has proved to be very effective: in 2013 practices targeted for enhanced support demonstrated the largest improvements in diabetes outcomes in the Borough.

\section{Virtual diabetes clinics}

Every practice in Lambeth is offered two virtual diabetes clinics per year, which consist of round table case discussions involving the Diabetes Intermediate Care Team and practice clinicians; one is attended by a hospital consultant or GPwSI and one is led by a diabetes specialist nurse. These can discuss problem cases, but often focus on patients with poor glycaemic control. The practice is provided with a clinical management plan for each case discussed, which often enables patients to continue to receive care at the surgery.

Virtual clinics have proved to be popular with primary care and are an excellent and practical way of delivering in-house diabetes education. In a case review conducted by the Diabetes Intermediate Care Team the average $\mathrm{HbA}_{1 \mathrm{c}}$ reduction for patients discussed in virtual clinics was $0.5 \%$.

\section{Telephone and email support to primary care}

Each Lambeth practice has a named specialist diabetes nurse. Truly excellent relationships have been built up over the past five years.
Figure 3. A session of Diabetes Education and Self Management for Ongoing and Newly Diagnosed type 2 diabetes patients (DESMOND)

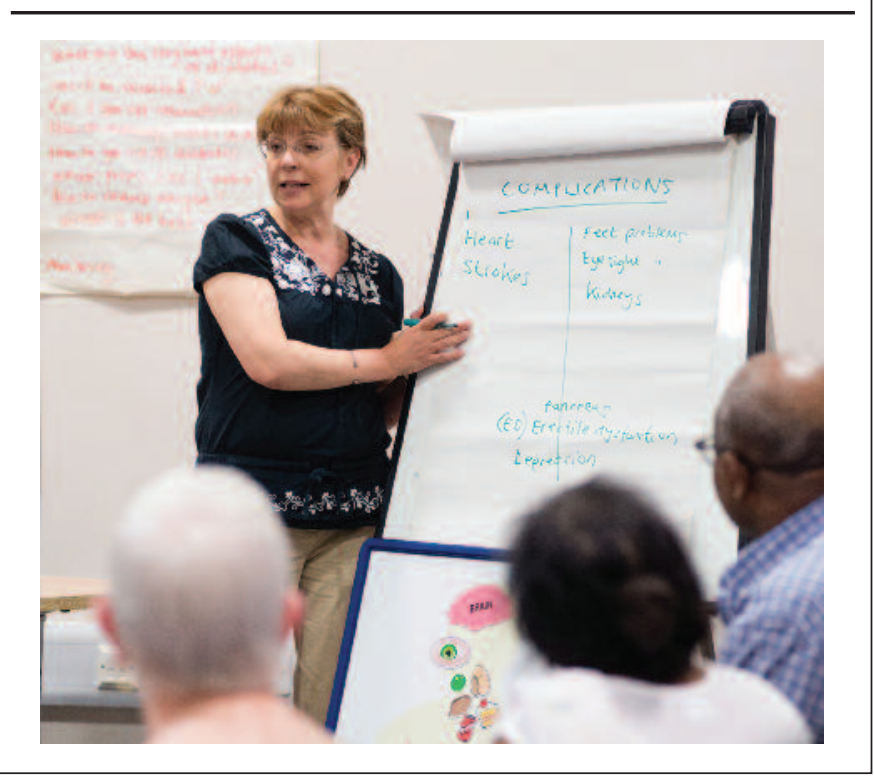

Practices know that they can call the team (or increasingly email a team member) if they require advice, whether urgent or routine.

\section{Insulin and GLP-1 start groups}

Most referrals to the community clinics are for people with poor glycaemic control and many will need to start an injectable therapy. Where possible, after a patient is seen in the community clinic they will be referred into a group injectable therapy session run by a diabetes specialist nurse. These groups are provided in each community clinic location every month and have a considerable educational focus covering lifestyle issues in addition to information about driving, travel, sick day rules and dose adjustment. After attending two group sessions, patients who have started injectable therapies are reviewed in the community clinic after 2-3 months.

\section{Professional education}

The Diabetes Intermediate Care Team provides regular educational sessions for local primary care clinicians. In particular an insulin management course called "Inform" has been developed over the past six years, with the aim of improving insulin management skills in local practices so that patients can be discharged back to their GP with good support from their practice. The Inform course was expanded in 2014 to include an online distance learning module created by King's College, London. After the course, learners are offered mentoring by a diabetes specialist nurse, who thus gains direct experience of starting patients on insulin in their own practice.

\section{Structured education for people with type $\mathbf{2}$ diabetes}

The team offers a range of structured education courses including DESMOND (Figure 3). Uptake is now amongst the best in London, although the conversion from "referral" to "attendance" remains a challenge. The team offer alternatives, to 
Figure 4. First outpatient appointments initiated by GPs for patients registered in Lambeth

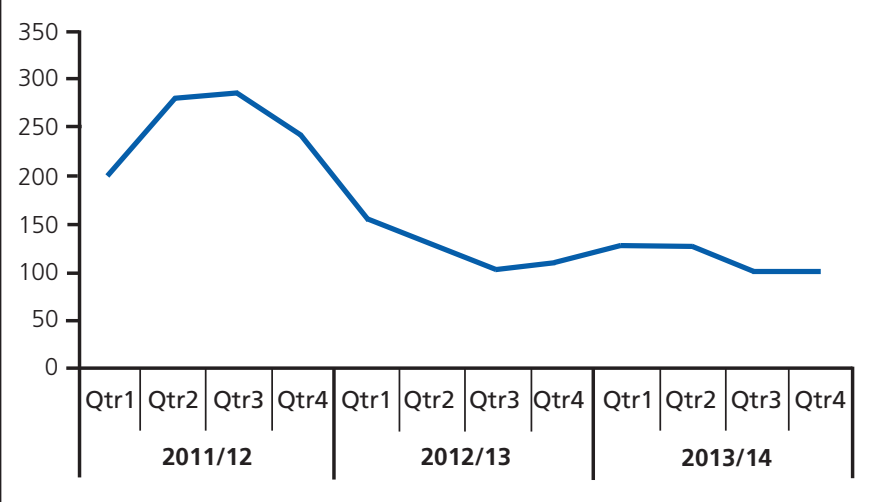

address this issue, including a 2-hour "Food and Diabetes" session for those unable to commit to six hours of DESMOND. Many local people do not have English as a first language, and the team offers an education course for people with type 2 diabetes and their families in Portuguese. Patients referred for education can also access the on-line "Help Diabetes" website developed at University College, London.

\section{Outcomes}

Has this service redesign been a success? The last four years have seen a steady reduction in referrals to hospital diabetes clinics (Figure 4), with a shift to the new community service, and also a reduction in local admissions due to diabetes (Figure 5). There has also been a sustained improvement in diabetes outcomes, with Lambeth moving from 26th to amongst the best for $\mathrm{HbA}_{1 \mathrm{c}}$ control among Clinical Commissioning Groups in London. There has been improved detection of diabetes in the Borough with a narrowing of the actual to expected prevalence gap and just under 3,000 more people diagnosed with type 2 diabetes.

\section{Future of the community diabetes service in Lambeth}

The model of diabetes care in Lambeth has proved a success in terms of service integration and patient outcomes and levels of patient satisfaction are very high. Capacity is now a challenge due to a high level of referrals, although the majority of patients will be seen within four weeks of referral by their GP. The service has found it challenging to achieve the 60\% discharge target set by the Clinical Commissioning Group. This largely relates to the increasing complexity of patients attending the community clinics and a lack of skills to manage complex insulin regimens in primary care. We hope that our insulin training programme will address this.

With the emergence of GP Federations in Lambeth, it seems
Figure 5. Emergency admissions for diabetes among patients registered in Lambeth and Southwark (Guy's and St Thomas' and King's College Hospital, London)
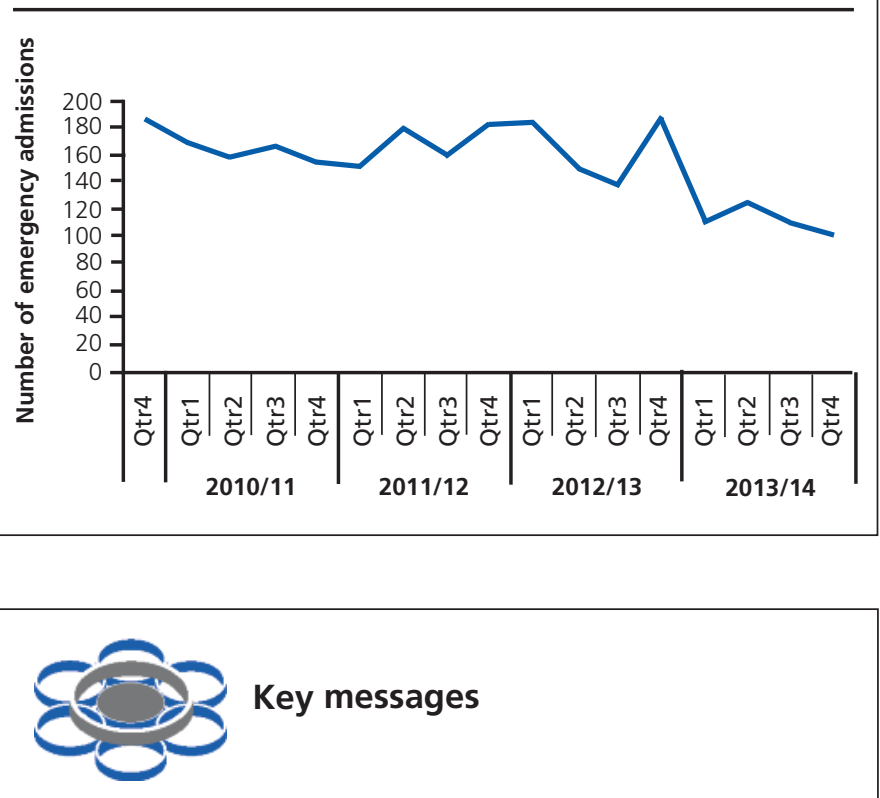

- Lambeth is an inner-city London borough with an ethnically and economically diverse population with a relatively high prevalence of diabetes

- A major reorganisation of care was undertaken in 2011, with a shift towards a highly integrated approach to multidisciplinary care delivered wherever possible for most patients in the community setting

- A range of initiatives to have been undertaken, overcome structural barriers to care, to promote better understanding of diabetes and self-management by patients and to enhance training and care provision from GP practices

- Improvements have occurred in local diabetes detection rates, diabetes outcomes, rates of referral to secondary care and rates of diabetes-related admissions

likely that some of the functions of the team could be provided by clusters of GP practices, such as the group injectable therapy sessions or patient education. However, the community clinics and the close working relationship between primary care teams and the Diabetes Intermediate Care Team have proved hugely popular with local general practice and the future of the service looks bright.

\section{Conflict of interest None. Funding None.}

